\title{
ECTOPARÁSITOS EN BUJURQUI (Cichlasoma amazonarum; PISCES: CICHLIDAE) CRIADOS EN ESTANQUES ARTIFICIALES
}

\author{
Ectoparasites in Cultivated Bujurqui (Cichlasoma amazonarum; Pisces: \\ Cichlidae) Reared in Fish Ponds
}

\author{
Juan Lo P. ${ }^{1}$, Amanda Chávez V. ${ }^{1,2}$, Guadalupe Contreras S. ${ }^{3}$, Nieves Sandoval C. ${ }^{4}$, \\ Cielo Llerena Z.$^{5}$
}

\section{Resumen}

Los objetivos del presente estudio fueron identificar y establecer las frecuencias de ectoparásitos en Cichlasoma amazonarum (Pisces: Cichlidae) criados en estanques en la zona de Iquitos, Perú, entre octubre y diciembre de 2004. Se colectaron ectoparásitos de tegumento y branquias en 227 peces. Los parásitos fueron teñidos con Hematoxilina de Delafield. La frecuencia de infecciones por ectoparásitos fue de $78.0 \pm 6.1 \%$ de monogeneos y $0.9 \%$ de maxillopoda (Crustacea: Branchiura). Dentro de los monogeneos, el $88.1 \%$ correspondió a parásitos de la familia Dactylogyridae, 6.2\% a la familia Gyrodactylidae y 5.7\% a infecciones mixtas de ambas familias. Los maxillopoda encontrados fueron de la especie Dolops geayi. El 80.2\% de los peces muestreados presentaron de uno a tres parásitos por individuo, lo que indica niveles bajos que no afectan la salud de los peces. No se encontró una correlación significativa entre las medidas biométricas de los peces con la infección de monogeneos. Los resultados del presente estudio indican que la presencia de monogeneos en cíclidos amazónicos peruanos es común.

Palabras clave: Dactylogyridae, Gyrodactylidae, monogeneos, Dolops geayi, Cichlasoma amazonarum, ectoparásito

\section{Abstract}

The aim of the present study was to identify ectoparasites and to establish the frequencies of infections in Cichlasoma amazonarum (Pisces: Cichlidae) raised in artificial ponds in Iquitos, Peru from October to December 2004. Ectoparasites collected from the skin and gills in 225 fish were stained using Delafield Hematoxiline. The frequency of ectoparasites was $78.0 \pm 6.1 \%$ monogeneans and $0.9 \%$ maxillopoda (Crustacea: Branchiura).

${ }^{1}$ Laboratorio de Microbiología y Parasitología Veterinaria, ${ }^{3}$ Estación Experimental del Centro de Investigación IVITA-Pucallpa, ${ }^{4}$ Laboratorio de Histología, Embriología y Patología Veterinaria, Facultad de Medicina Veterinaria, Universidad Nacional Mayor de San Marcos, Lima

${ }^{2}$ E-mail: achavezvg@gmail.com

${ }^{5}$ Facultad de Veterinaria y Zootecnia, Universidad Peruana Cayetano Heredia, Lima 
Among monogenean infections, 88.1\% corresponded to parasites of Dactylofyridae family and $6.2 \%$ of Gyrodactylidae family, whereas 5.7\% to mixed infections of both families. All maxillopoda found were Dolops geayi. One to three parasites per individual were found in $80.2 \%$ of the sampled fish, indicating of low parasite burden, which may no affect fish health. There was no significant correlation between biometric measurements and monogeneans infection in C. amazonarum. This study could indicate that monogeneans in Peruvian Amazon cichlids are common.

Keywords: Dactylogyridae, Gyrodactylidae, monogenean, Dolops geayi, Cichlasoma amazonarum, ectoparasites

\section{INTRODUCCIÓN}

Los ectoparásitos se ubican en el tegumento, branquias, ojos, narinas y boca de los peces hospedadores (Roberts, 1981; Eiras et al., 2000), causando lesiones por su sola presencia física, por secreciones químicas o excreciones o por el modo de alimentarse (Thatcher, 1991), así como por la transmisión de bacterias, parásitos y virus (Noga, 1996). Entre los ectoparásitos más importantes resaltan los monogeneos y los crustáceos.

Los monogeneos parasitan peces marinos y de aguas continentales (Merck, 2000), ocasionando cuantiosas pérdidas económicas en los sistemas de producción piscícola. Se les considera uno de los parásitos de mayor relevancia por las elevadas tasas de mortalidad que pueden ocasionar, especialmente en algunos cíclidos como la tilapia (Flores y Flores, 2003).

Los monogeneos son gusanos planos hermafroditas de ciclo corto y directo (Thatcher, 1991; Vidal et al., 2002), de gran especificidad de hospedero (Sasal et al., 1999, 2004; Šimková et al., 2001, 2005) debido a que una especie solo afecta a una o varias especies de peces relacionadas (Carnevia, 1993). Su cuerpo tiene una parte anterior denominada prohaptor, que contiene las manchas oculares, boca, glándulas de fijación, sistema digestivo, sistema reproductor y aparato excretor; y una parte posterior representada por el opistohaptor, órgano adhesivo discoidal aplanado que contiene ganchos y ba- rras (Vidal et al., 2002; Flores y Flores, 2003) con los que se fijan a las branquias o al tegumento del hospedador ocasionándole lesiones.

Las familias más importantes dentro del orden Monogenea desde el punto de vista patológico es la Gyrodactylidae, conocida como gusanos de piel y la familia Dactylogyridae, conocida como gusanos de branquias (Roberts, 1981), las que tienen un tipo de reproducción vivípara y ovípara, respectivamente.

Las fuentes de infección son otros peces parasitados, el fondo del estanque donde completan su desarrollo los huevos de algunos miembros de la familia Dactylogyridae, y el agua del estanque debido a la presencia de larvas oncomiracidios de nado libre. La transmisión es por contacto directo de pez a pez (Reed et al., 2002), favorecida por la proximidad de los hospederos de la misma especie, como sucede en cultivos intensivos (Huet, 1978; Reichenbach-Klinke, 1982). El diagnóstico de parásitos de la familia Gyrodactylidae se hace mediante el raspado del tegumento y observación al microscopio, y en el caso de parásitos de la familia Dactylogyridae a través de la observación de gusanos adheridos a los filamentos branquiales (Carnevia, 1993).

La identificación de los monogeneos se basa en la morfología de las estructuras esclerotizadas del haptor o del complejo copulatorio (Reichenbach-Klinke, 1982; Thatcher, 1991; Kritsky et al., citado por Eiras et al., 2000) unida a la marcada especifici- 


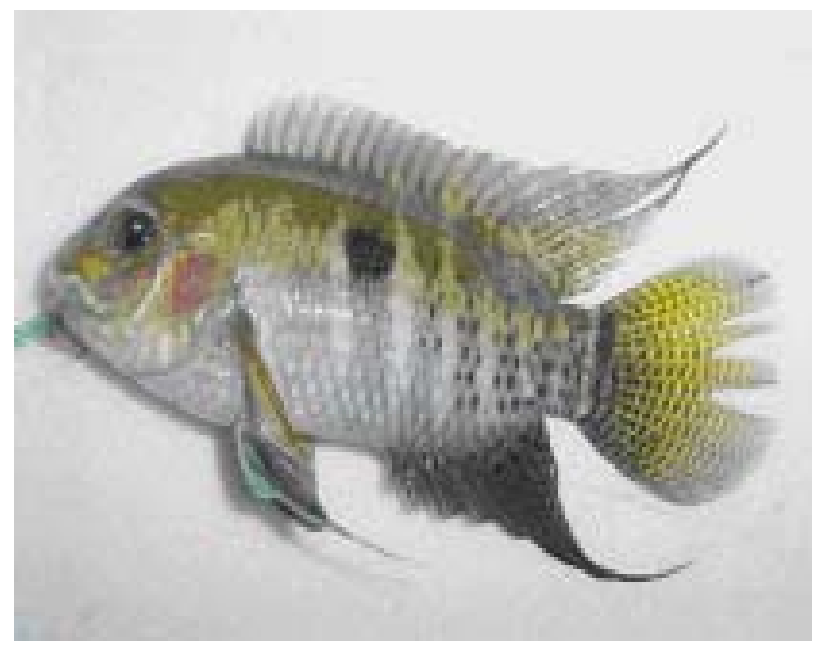

Figura 1. Bujurqui (Cichlasoma amazonarum)

dad de hospedadores (Flores y Flores, 2003) que es relevante para la caracterización de familias y géneros (Eiras et al., 2000); y se corrobora empleando claves de identificación, como las publicadas por Yamaguti (1963) y Thatcher (1991).

Los crustáceos parásitos de peces de agua dulce, salada y salobre más comunes son los Argulus sp. y Dolops sp. (Eiras et al., 2000). Estos branchiuridos presentan sexos separados y cuerpos aplanados dorsoventralmente, adaptados para el movimiento rápido sobre la superficie cutánea (Merck, 2000); su ciclo de vida es de 30 días o más, dependiendo de la especie y de la temperatura medioambiental (Noga, 1996). La transmisión es directa puesto que pueden nadar activamente y fijarse al pez hospedador, así como cambiar de hospedador en repetidas oportunidades (Roberts, 1981).

En el Perú, el cultivo de peces amazónicos ornamentales y de consumo se encuentra susceptible a los efectos nocivos de estos parásitos (Conroy, 2000) debido a la carencia de facilidades para el diagnóstico y falta de investigación en esta área. En el caso de bujurqui [Cichlasoma amazonarum (Kullander, 1986)] (Fig. 1), un cíclido nativo de la cuenca Amazónica, que puede repro- ducirse en estanques de cultivo de manera continua (Silva et al,. 2004), tanto para el consumo humano como para forraje vivo en cultivo de Arapaima gigas (Alcántara y Guerra, 1992; Alcántara et al., 2004; Panduro y Portugal, 2004; Silva et al., 2004), así como en cultivos asociados con Cichla ocellaris (Molnar et al., 2001) y como pez ornamental para el mercado internacional.

El presente estudio se llevó a cabo con el fin de identificar y establecer las frecuencias de ectoparásitos presentes en Cichlasoma amazonarum mantenidos en cautiverio bajo un sistema de cultivo semicomercial en la zona de Iquitos, Perú.

\section{Materiales y Métodos}

\section{Zona del Estudio}

El estudio se realizó en las instalaciones del Proyecto Las Flores "Ornamental Amazon Fish Aquarium” (OAFA), Iquitos. Las muestras se procesaron en el mismo lugar, en la Estación Experimental del Centro de Investigación IVITA-Iquitos y en el Laboratorio de Parasitología de la Facultad de Medicina Veterinaria de la Universidad Nacional Mayor de San Marcos, Lima. 


\section{Crianza del Cichlasoma amazonarum}

Ejemplares juveniles de C. amazonarum fueron capturados en riachuelos cercanos a la ciudad de Iquitos, acondicionados en acuarios, y mantenidos por 60 días con alimento peletizado para peces ornamentales. Luego se seleccionó los mejores ejemplares para formar el plantel de reproductores, tomando en cuenta características como velocidad de crecimiento, coloración llamativa y buena conformación corporal.

Los reproductores se colocaron en un estanque rectangular de paredes de ladrillo $\mathrm{y}$ cemento de $14.7 \mathrm{~m}^{3}$ (4.2 x $\left.3.5 \times 1 \mathrm{~m}\right)$, con entrada y salida constante de agua, proveniente de los excesos de agua del manejo de las instalaciones de OAFA. El estanque de cultivo fue ambientado con lechuga de agua (Pistia stratiotes) para brindar protección contra el sol y depredadores. Los peces se alimentaron ad libitum con guppy (Poecilia reticulata), además de fitoplancton, zooplancton e insectos presentes en el agua.

El agua del acuario tenía un $\mathrm{pH}$ de 8.07 (8.0-8.2), temperatura de de 25 a $26^{\circ} \mathrm{C}$, una conductividad de $855 \mu$ s (843-867 $\mu$ s), oxígeno disuelto $8 \mathrm{mg} / \mathrm{l}$, nitritos $0.4 \mathrm{mg} / \mathrm{l}$, nitratos 5 $\mathrm{mg} / \mathrm{l}$, y dureza total $4.67^{\circ} \mathrm{DH}$. Estas observaciones fueron realizadas al medio día. El estanque tenía una densidad de 34.0 peces $/ \mathrm{m}^{3}$.

Los peces fueron mantenidos en el estanque durante 15 meses previos al muestreo. Durante los primeros meses se observó el comportamiento de alimentación, formación de parejas, defensa de territorios y cortejo. Asimismo, se comprobó la presencia de alevinos de pocos centímetros, indicando reproducción exitosa de C. amazonarum en el estanque.

\section{Tamaño Muestral}

Se determinó mediante la tabla de Simon y Schill (Eiras et al., 2000) para un nivel de confianza de 95\%, con la certeza de detectar por lo menos un espécimen parasitado y pre- sumiendo una prevalencia del $1 \%$. Se obtuvo un número de muestra mínima de 224, pero se trabajó con 227 peces tomados al azar de un estanque de 500 ejemplares de $C$. amazonarum de diferentes tallas.

\section{Obtención y Manejo de Muestras}

El muestreo se realizó en octubre, noviembre y diciembre del 2004, evaluando una cantidad similar de peces por mes. Los peces se sacrificaron mediante una perforación a la altura de la primera vértebra, siguiendo la recomendación de Eiras et al. (2000). Se pesaron y se midió la longitud total (cabeza a la aleta caudal) y longitud estándar (cabeza al final de la columna vertebral).

Para la obtención de las muestras se modificó el trabajo de Eiras et al. (2000). Se realizó una observación macroscópica general evitando la desecación de los tejidos rociando agua constantemente. Se hicieron raspados del tegumento, aletas y lados de la cabeza del pez con una hoja de bisturí en sentido anteroposterior. Los raspados se colocaron en láminas portaobjetos con una gota de agua, y se observaron en un microscopio óptico. Los parásitos encontrados se fijaron en alcohol al 70\%.

Además, se realizó el examen de las branquias. Se retiraron los óperculos y se observó primeramente in situ y luego cada arco branquial en una placa petri con agua con un microscopio óptico o un microscopio estereoscópico. Los parásitos encontrados fueron fijados en alcohol al 70\%. Asimismo, el agua de hidratación de las branquias y de las zonas de raspado se revisó con un microscopio estereoscópico, para rescatar parásitos que se hubieran podido desprender del hospedero.

En la coloración de los monogeneos y branchiuridos se utilizó la Hematoxilina de Delafield (INS, 2003). En la clasificación de los monogeneos se emplearon los trabajos de Yamaguti (1963), Thatcher (1991) y Noga (1996), y para los crustáceos se utilizó el tra- 
Cuadro 1. Prevalencias de ectoparásitos hallados en 227 ejemplares de Cichlasoma amazonarum mantenidos en cautiverio en Iquitos, Perú (2004)

\begin{tabular}{llcc}
\hline Grupos & Peces parasitados & Prevalencia \pm I.C. ${ }^{1}(\%)$ \\
\hline Monogeneo & & 177 & $78.0 \pm 6.1$ \\
& Dactylogyridae & 156 & $88.1 \pm 4.8$ \\
& Gyrodactylidae & 11 & 6.2 \\
& Ambas & 10 & 5.7 \\
Maxillopoda & & 0.9 \\
& Dolops geayi & 2 & $78.4 \pm 6.0$ \\
\hline Total de ectoparásitos & $178^{2}$ & \\
\hline${ }^{1}$ Intervalo de confianza al 95\%. &
\end{tabular}

bajo de Thatcher (1991). Además, se enviaron fotografías a especialistas del Instituto Nacional de Pesquisas de la Amazonía de Brasil y del Instituto de Parasitología de la Academia de Ciencias de la República Checa.

Los resultados se expresaron en porcentajes con intervalos de confianza del 95\%. Se utilizó el programa estadístico Stata ${ }^{\circledR}$ v. 8.0. Se hizo un análisis de regresión para determinar la posible asociación entre las medidas biométricas y la carga parasitaria, empleando los programas Excel y SPSS 11.

\section{Resultados}

No se observaron lesiones macroscópicas ni signos clínicos que indiquen un desmedro en la salud y condiciones de los peces.

El Cuadro 1 muestra una frecuencia de $78.4 \pm 6.1 \%$ de ectoparásitos en $C$. amazonarum donde la mayor parte fueron monogeneos (78.0 $\pm 6.1 \%, 177 / 227)$ y muy pocos fueron del tipo crustáceo (Dolops geayi, Fig. 2). Así mismo, dentro de los monogeneos, la mayor parte de los parásitos encontrados fueron de la familia Dactylogyridae (88.1 \pm 4.8\%, 156/177, Fig. 3a) y sólo el 6.2\% (11/177) de la familia Gyrodactylidae (Fig. 3b), en tanto que el resto presentaron infecciones mixtas de ambas familias $(5.7 \%)$. Por otro lado, se encontró entre 1 a 3 monogeneos en el $80.2 \%$ de los peces parasitados, mientras que el 19.8 tuvo $\geq 4$ parásitos.

El análisis de regresión para determinar una posible asociación entre longitud total, longitud estándar o peso con la carga parasitaria indicó una relación no significativa.

\section{Discusión}

Se sabe que los parásitos con ciclos biológicos directos suelen ser patógenos de importancia para peces de cultivo (Merck, 2000). Por otro lado, los peces silvestres en condiciones naturales presentan parásitos en escasas cantidades, pudiendo ser considerado como un hallazgo normal que raramente causa problemas patológicos (Brown, 2000).

La frecuencia de $C$. amazonarum con ectoparásitos (78.4 $\pm 6.1 \%)$ es una cifra elevada si se le compara con los resultados en cíclidos del sureste de México (Jiménez, 1993) y de Thilakaratne et al. (2003). Estos últimos hallaron $45.3 \%$ de parasitados en 13 espe- 


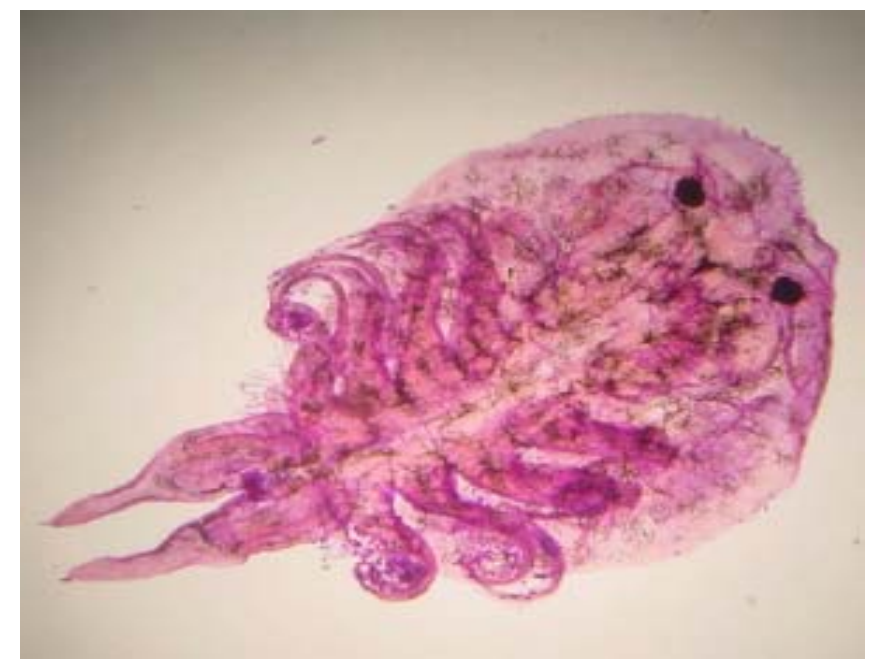

Figura 2. Crustáceo branchiurido Dolops geayi

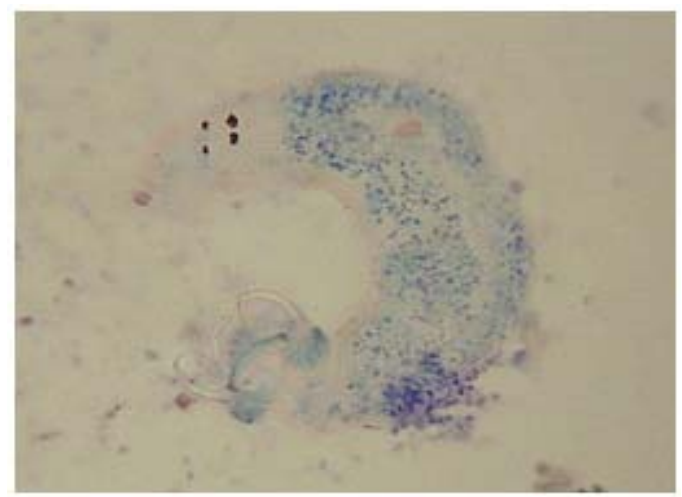

(a)

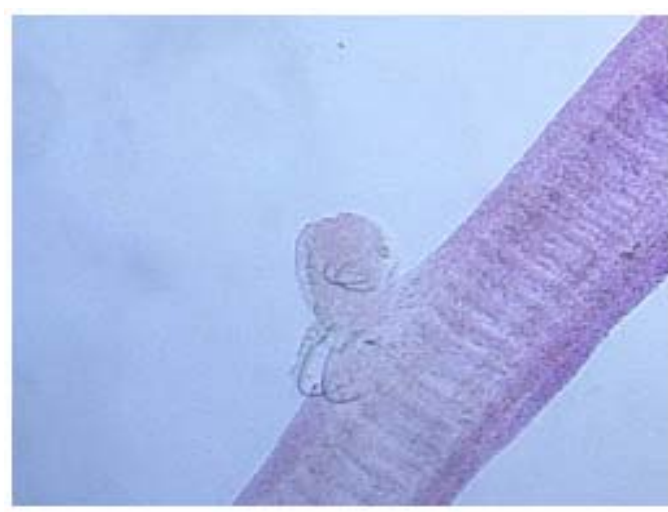

(b)

Figura 3. Monogeneo. (a) Individuo de la Familia Dactylogiridae; (b) Individuo de la Familia Gyrodactylidae

cies de peces ornamentales en 26 granjas de Sri Lanka. La elevada prevalencia de ectoparásitos del presente estudio podría deberse a una exacerbación en la reproducción y transmisión de parásitos ante una alta densidad de peces en el estanque (Huet, 1978), así como por las condiciones favorables del medio de cultivo para el caso de parásitos de la familia Dactylogyridae (Roberts, 1981).

Los hallazgos del presente estudio indican la presencia de monogeneos en cíclidos amazónicos peruanos; sin embargo, la baja carga parasitaria no ocasionaría lesiones macroscópicas ni signos clínicos que puedan ser observados durante la recolección de muestras.

El predominio de la familia Dactylogyridae sobre la familia Gyrodactylidae se podría explicar por las condiciones de cultivo que favorecen su reproducción ovípara (Roberts, 1981), y por una posible "resistencia” de la epidermis del C. amazonarum a las infecciones de monogeneos; menguando así la reproducción de la familia 
Gyrodactylidae, obligando a estos parásitos a ubicarse en las branquias, donde tiene menor especialización para fijarse a la mucosa que la familia Dactylogyridae (Thatcher, 1991).

Es posible que el ciclo de vida directo de los monogeneos y las condiciones del cultivo intensivo de C. amazonarum en los estanques hayan favorecido la transmisión, reproducción y supervivencia de los parásitos llegando a niveles de riesgo; condiciones que pueden ocasionar brotes agudos de infecciones parasitarias con lesiones graves e infecciones secundarias a causa de bajas inmunitarias en los hospedadores (Carnevia, 1993). Adicionalmente, los cambios bruscos en las características físico-químicas del agua (Brown, 2000) pueden causar mortalidad y morbilidad en los peces de cultivo.

La prevalencia encontrada del crustáceo branchiurido $D$. geayi en $C$. amazonarum fue bastante más baja (0.9\%) en comparación con el 30\% reportado en pirañas en cautiverio y en ambientes naturales del Pantanal, Brasil (Thatcher, 1991); por lo que se presume que las condiciones de crianza del C. amazonarum no hayan ofrecido las condiciones adecuadas para su desarrollo. Sin embargo, el hallazgo de D. geayi puede considerarse como el primer reporte de $C$. amazonarum en el Perú.

La dominancia numérica de monogeneos sobre crustáceos parásitos puede explicarse por su alta especificidad (Sasal et al., 1999, 2004; Šimková et al., 2001, 2005), así como a la puesta de huevos o larvas oncomiracidios directamente en las branquias de los peces hospedadores por parte de los monogeneos.

El hallazgo de monogeneos de las familias Gyrodactylidae y Dactylogyridae, así como del crustáceo parásito Dolops geayi en $C$. amazonarum mantenidos en estanques de crianza comercial implica el ingreso de peces portadores a causa de un mal manejo sanitario y a las características de reproduc- ción y fácil traspase de los parásitos. A partir de esto, se confirma que es posible las infestaciones de ambas familias de monogeneos en peces de acuario, y no sólo de la Familia Gyrodactylidae como se afirma en otros estudios (Carnevia, 1993).

La exportación de peces ornamentales amazónicos portadores de ectoparásitos puede producir su diseminación (Flores y Flores, 2003), pudiendo causar infecciones severas y pérdidas importantes, llegando a establecerse en ambientes naturales afectando a peces silvestres (Roberts, 1981). Para evitar estos efectos, se deben realizar tratamientos preventivos rutinarios como medida de control en acuarios de cuarentena previos a la movilización de estas especies de su sitio de origen.

\section{Literatura Citada}

1. Alcántara F, Guerra H. 1992. Cultivo de paiche, Arapaima gigas, utilizando bujurqui, Cichlasoma bimaculatum, como presa. Folia Amazónica 4(1): 133144

2. Alcántara F, Tello S, Chávez C, Rodríguez L, Kohler C, Camargo W. 2004. Cultivo de paiche, Arapaima gigas en estanque de productores de la carretera Iquitos - Nauta. [Internet], [25 enero 2005]. Disponible en: http:// www.siamazonia.org.pe/ Detalleventos/ S e t i e m b r e \% $2020002 /$ Curso_intern_acuicultura/ Bibliogr_adicional/Paiche.pdf

3. Brown L. 2000. Acuicultura para veterinarios. Producción y clínica de peces. España: Acribia. 460 p.

4. Carnevia D. 1993. Enfermedades de los peces ornamentales. Buenos Aires: Ed Agrovet. 320 p.

5. Conroy G. 2000. Principales enfermedades en peces de aguas tropicales. En: Libro de Resúmenes del II Seminario Internacional del FONDEPES Acuicultura: 
Actividad Prioritaria del Tercer Milenio. Lima: FONDEPES. p 25.

6. Eiras J, Takemoto R, Pavanelli G. 2000. Métodos de estudio y técnicas laboratoriales en parasitología de peces. España: Acribia. 142 p.

7. Flores J, Flores R. 2003. Monogeneos, parásitos de peces en México: estudio recapitulativo. Téc Pec Méx 41(2): 175192.

8. Huet M. 1978. Tratado de piscicultura. $2^{\mathrm{da}}$ ed. España: Mundiprensa. 784 p.

9. [INS] Instituto Nacional de Salud. 2003. Manual de procedimientos de laboratorio para el diagnóstico de los parásitos intestinales del hombre. Serie de Normas Técnicas. 90 p. [Internet], [20 diciembre 2010]. Disponible en: http:// bvs.minsa.gob.pe/local/INS/ 165_NT37.pdf

10. Jiménez M. 1993. Fauna helmintológica de Cichlasoma fenestratum (Pisces: Cichlidae) del lago de Catemaco, Veracruz, México. Anales Inst Biol Univ Auton México, Ser Zool 64(1): 75-78.

11. Kullander S. 1986. Cichlid fishes of the Amazon River drainage of Peru. Stockholm: Swedish Museum of Natural History. $431 \mathrm{p}$.

12. Merck 2000. El Manual Merck de Veterinaria. $5^{\mathrm{a}}$ ed. Barcelona: Océano. 2558 p.

13. Molnar J, Alcántara F, Tello S. 2001. Identifying goals and priorities of fish farmers in the Peruvian Amazon. In: Gupta A, Mc Elwee K, Burke D, Burright J, Cummings X, Egna H (eds). XVIII Annual Technical Report. Pond Dynamics / Aquaculture. Oregon: CRSP. p 131-134.

14. Noga. 1996. Fish disease: diagnosis and treatment. USA: Mosby Year Book. 367 p.

15. Panduro G, Portugal V. 2004. Evaluación del crecimiento de paiche (Arapaima gigas) alimentado con peces vivos y muertos durante la fase de engorde en ambientes controlados, Iquitos (Perú). CIVA 2004. p 808-813. [Internet], [05 mayo 2005]. Disponible en: http://www.civa2004.org
16. Reed P, Francys-Floyd R, Klinger $R$. 2002. Monogenean parasites of fish. FA28. Institute of Food and Agricultural Sciences, University of Florida. [Internet], [15 mayo 2005]. Disponible en: http://edis.ifas.ufl.edu/pdffiles/FA/ FA03300.pdf

17. Reichenbach-Klinke H. 1982. Enfermedades de los peces. $2^{\text {a }}$ ed. España: Acribia. 524 p.

18. Roberts R. 1981. Patología de los peces. Madrid: Mundi-Prensa. $366 \mathrm{p}$.

19. Sasal P, Trouve S, Müller-Graf C, Morand S. 1999. Specificity and host predictability: a comparative analysis among monogenean parasites of fish. J Anim Ecol 68(3): 437-444.

20. Sasal P, Desdevises Y, Durieux E, Lenfant P, Romans P. 2004. Parasites in marine protected areas: success and specificity of monogeneans. J Fish Biol 64: 370-379.

21. Silva M, Alcántara F, Del Águila M. 2004. Reproductive biology of the bujurqui Cichlasoma amazonarum (Kullander, 1983) in controlled environments. In: Roubach R, Carvalho L, Mac Kinlay D (eds). VI International Congress on the Biology of Fish: Fish culture performance in the tropics. American Fisheries Society. [Internet], [23 agosto 2005]. Disponible en: http:// www-heb.pac.dfo-mpo.gc.ca/congress/ 2004/Culture/35SilvaRepro.doc

22. Šimkova A, Desdevises Y, Gelnar M, Morand S. 2001. Morphometric correlates of host specificity in Dactylogyrus species (Monogenea) parasites of European Cyprinid fish. Parasitology 123: 169-177.

23. Šimkova A, Jardovský J, Koubková B, Barus V, Prokeš M. 2005. Associations between fish reproductive cycle and the dynamics of metazoan parasite infection. Parasitol Res 95(1): 65-72.

24. Tantaleán M, Sarmiento L, Huiza A. 1992. Digeneos (Trematoda) del Perú. Bol Lima 80: 47-84.

25. Thatcher V. 1991. Amazon fish parasites. Amazoniana 11(3-4): 263-571. 
26. Thilakaratne I, Rajapaksha G, Hewakopara R, Rajapakse R, Maizal A. 2003. Parasitic infections in fresh water ornamental fish in Sri Lanka. Dis Aquat Organ 54: 157-162.

27. Vidal V, Aguirre M, Scholz T, Gonzáles D, Mendoza E. 2002. Atlas de los helmintos parásitos de cíclidos de México. México: Instituto Politécnico Nacional. 183 p.

28. Yamaguti S. 1963. Systema helminthum. Vol. IV. Monogenea and Aspidocotylea. New York: Interscience Publishers. 699 p. 